\title{
Herramientas metodológicas para la gestión del patrimonio intangible. El caso del municipio de Olavarría, Buenos Aires, Argentina
}

Methodological Tools for the Management of Intangible Heritage. The Case of the Municipality of Olavarría, Buenos Aires, Argentina

\author{
Mercedes Mariano, María Luz Endere y Carolina I. Mariano \\ Universidad Nacional del Centro de la Provincia de Buenos Aires. \\ Patrimonia, Incuapa (UE-Conicet-Unicen) \\ mercedes.mariano@gmail.com \\ mendere@soc.unicen.edu.ar \\ caro.mariano@hotmail.com
}

\section{$\overline{\text { RESUMEN }}$}

En los últimos años, la salvaguarda del patrimonio cultural intangible ha comenzado a ocupar un lugar destacado en la agenda de los Estados nacionales, aunque en Argentina aún no se han unificado criterios para su gestión. En este artículo se presentan los lineamientos generales para el desarrollo de una propuesta de registro del patrimonio cultural intangible, tomando como caso de estudio el municipio de Olavarría, provincia de Buenos Aires, Argentina. Para ello se tuvieron en cuenta experiencias previas de inventarios efectuadas en el país y en el exterior. A través de este trabajo, se pretende hacer un aporte al desarrollo de metodologías aplicables a la gestión del patrimonio cultural intangible, que contribuyan a incrementar su visibilización y valoración social.

Palabras clave: patrimonio intangible, gestión, metodología de inventario, SIG. $\overline{\text { ABSTRACT }}$

In recent years, the safeguarding of intangible cultural heritage has begun to occupy a key position in the agenda of national States, although in Argentina there are still no unified criteria for its management. This paper presents general guidelines for the development of a proposal for the management of intangible cultural heritage, taking the municipality of Olavarría, Buenos Aires province, Argentina as a case study. In this paper, previous inventories carried out in the country and abroad were also taken into account. Through this analysis is intended to contribute to the development of new methodologies for the management of intangible cultural heritage which help to increase its visibility and social valuation.

Keywords: intangible heritage, management, inventory methods, GIS. 


\section{Introducción}

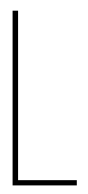

a gestión del patrimonio cultural no solo consiste en identificar, proteger y presentar al público las expresiones de la cultura material e inmaterial de los pueblos en un contexto de cambio permanente, sino que implica, a su vez, un proceso de selección, jerarquización y control de las diversas manifestaciones culturales en clasificaciones que suelen delimitar el carácter de tal gestión. La patrimonialización cultural no es inocente ni unívoca, pues no es un secreto que la idea de patrimonio es una construcción social de Occidente que ha movilizado y moviliza, aún hoy, intereses políticos y económicos (Salge 2014). En su nombre se han legitimado tanto la administración de las identidades y los Estados nacionales como los discursos de resistencia y las iniciativas locales para el "mejoramiento de las condiciones de vida de comunidades tradicionalmente excluidas” (Salge 2014, 4). Así pues, el patrimonio cultural inmaterial parece ser, también, un campo propicio para la exaltación de territorios y grupos periféricos y pobres. En el marco de los proyectos de integración nacional de varios países, la prioridad dada a las manifestaciones de carácter popular y festivo en peligro de extinción tiene que ver con el carácter atractivo que es exacerbado por los agentes del espectáculo y el turismo. Se trata de procesos que, más bien, despolitizan las expresiones culturales que celebran y terminan por legitimar lugares y grupos centrales (Lacarrieu 2010).

En este contexto, la ratificación de la Convención para la Salvaguarda del Patrimonio Cultural Inmaterial (Unesco 2003) por parte de diferentes países ha significado un desafío para la generación de políticas, estrategias y herramientas de gestión adecuadas para salvaguardar el patrimonio cultural intangible (en adelante, PCI). La mayoría de los países que ratificaron esa convención cuentan con pocos antecedentes de registro y clasificación de los bienes inmateriales, con excepción de algunas expresiones que han sido de particular interés en determinadas regiones. Por sus características, el PCI resulta mucho más complejo de clasificar. Además, la dificultad de su gestión radica en la naturaleza y las múltiples problemáticas que emergen a partir de su conceptualización, así como 
en la enorme diversidad y cantidad de expresiones y manifestaciones que lo componen, y de los grupos, comunidades e individuos involucrados.

En años recientes, varios países latinoamericanos han comenzado a establecer políticas de protección empleando modelos clasificatorios y diversas metodologías para la creación de herramientas de gestión, como ha sucedido particularmente en Brasil y Colombia. En el caso de Argentina, por tratarse de un país federal, las provincias tienen las facultades de dictar sus propias normas y políticas de protección, aunque subordinándose al marco legal federal. Desde la Secretaría de Cultura de la Nación, se está trabajando en la elaboración de políticas de gestión del PCI a ser implementadas de manera conjunta con todas las provincias. No obstante, todavía no se han hecho públicos los criterios en que se basan ni se ha procedido a su implementación. Paralelamente, diferentes provincias han puesto en marcha sus propios programas, los cuales han dado lugar a resultados diversos que se volcaron en atlas e inventarios dinámicos, entre otros. A través de estas iniciativas, sin embargo, no se suele abordar la totalidad de las manifestaciones posibles del PCI provincial, sino aquellas que son consideradas más relevantes o representativas (e. g., la Ciudad Autónoma de Buenos Aires solo se ha ocupado del inventario de fiestas, celebraciones y rituales). A nivel local, los municipios cuentan con variados patrimonios que, en general, han sido escasamente protegidos.

En consecuencia, podría afirmarse que gran parte del PCI de Argentina se encuentra invisibilizado y, por ende, no es mayormente conocido ni valorado. Es por ello que, sin perder de vista las observaciones antes efectuadas en relación con las múltiples miradas, voces e intereses que intervienen en la selección y jerarquización de los elementos que integran el PCI, se considera necesario generar instrumentos de gestión que conduzcan a salvaguardar el patrimonio, comenzando por el levantamiento de información de base. En este sentido, el objetivo de este trabajo consiste en desarrollar y discutir un marco metodológico que genere herramientas eficaces para la gestión del PCI a nivel local. Para ello, se tomará como caso de estudio el municipio de Olavarría, ubicado en el centro de la provincia de Buenos Aires.

Cabe señalar que este caso está siendo usado como una experiencia piloto, susceptible de ser replicada en otros municipios de la provincia y del país. Se eligió trabajar a nivel de la gestión local, por ser esta la escala en la que se definen las políticas de ordenamiento y gestión territorial en Argentina. Esta propuesta es parte de un trabajo de investigación desarrollado por el Programa Patrimonia (Estudios Interdisciplinarios de Patrimonio), uno de cuyos objetivos a largo plazo es la creación de un sistema de información que reúna datos relativos a las 
expresiones del PCI del centro de la provincia de Buenos Aires y que se integrará al que se está desarrollando desde el 2009 sobre el patrimonio cultural material ${ }^{1}$.

\section{Antecedentes en torno a la conceptualización del patrimonio cultural intangible}

La diversidad de expresiones culturales y la multiplicidad de sus significaciones son elementos presentes y constitutivos en la actual definición de patrimonio cultural que es postulada a nivel internacional. Lejos quedó aquella primera conceptualización que lo vinculaba con un conjunto de bienes materiales con un valor intrínseco al margen de los conflictos y dinámicas sociales. Ahora no solo se identifican las costumbres, expresiones y sistemas de creencias, sino que se amplía el enfoque al reconocer tanto los aspectos materiales como los inmateriales de las culturas (García Canclini 1999; Prats 2000, 2007; Rosas Mantecón 1998; Unesco 2003).

La relación entre la antropología y el patrimonio cultural remite a discusiones teóricas sobre paradigmas clásicos estructural-funcionalistas y culturalistas de la disciplina, los cuales han influido en la dimensión simbólica e identitaria del patrimonio cultural y en sus lógicas de activación. Ejemplos de ello son los significados subyacentes de nociones como rescate y tradición, tan prominentes en la producción etnográfica (principalmente textual), y el uso del tiempo y el espacio como elementos distintivos de una alteridad cultural pretendidamente congelada y circunscrita. Por otra parte, la concepción de la cultura como entramado de símbolos, ideas y valores en continua construcción ha guiado la ampliación del concepto de patrimonio cultural y, con ello, el repertorio de conocimientos, datos y metodologías. En América Latina, el replanteamiento crítico del tema fue impulsado por antropólogos como García Canclini (1999) y Bonfil Batalla (1991), lo cual promovió la reflexión en los terrenos de la antropología aplicada (Rosas Mantecón 1998,4) e incorporó la dimensión del conflicto en el análisis de los patrimonios culturales. García Canclini (1999) explica que los discursos actuales referidos al patrimonio cultural comenzaron a mostrar

1 El grupo Patrimonia ha desarrollado el Sistema de Información del Patrimonio Arqueológico Bonaerense (Sipab) (Mariano 2013). 
cambios en tres sentidos principales: en primer lugar, se reconoció que el patrimonio no incluye solo la herencia de cada pueblo o las expresiones "muertas” de su cultura (sitios arqueológicos, arquitectura colonial, objetos antiguos en desuso), sino también los bienes culturales que se reconocen en un espacio y en un tiempo actual (nuevas artesanías, lenguas, conocimiento, tradiciones). En segundo lugar, la política patrimonial de la conservación y administración de lo producido en el pasado se extendió a los usos sociales que relacionan esos bienes con las necesidades contemporáneas de las mayorías. Por último, frente a una selección que privilegiaba los bienes culturales producidos por las clases hegemónicas, se reconoció que "el patrimonio de una nación también está compuesto por los productos de la cultura popular: música indígena, escritos de campesinos y obreros, sistemas de autoconstrucción y preservación de los bienes materiales y simbólicos elaborados por grupos subalternos” (García Canclini 1999, 16-17). Este camino permitió no solo plantear que es imposible centrar los análisis de los bienes culturales aislados de su proceso de producción y circulación social, sino también resaltar la importancia de los actores en la construcción y valoración de los patrimonios culturales. De este modo, se reconoce el patrimonio en tanto construcción social y se lo entiende como ámbito de enfrentamientos y negociaciones sociales, como medio para reproducir identidades y diferencias sociales (Rosas Mantecón 1998, 5).

El fenómeno patrimonial comienza a ser entendido entonces como un campo cultural donde interactúan la producción social, la distribución y el uso de aquellos bienes que se caracterizan como patrimoniales. Un factor determinante para definir el patrimonio cultural es su capacidad para representar simbólicamente una identidad (Prats 2007; Rosas Mantecón 1998). Tanto el patrimonio como la identidad se caracterizan por su dinamismo y flexibilidad ante contextos sociales y políticos. Así, la producción simbólica de ciertas manifestaciones convertidas en bienes culturales y resignificadas dentro de discursos de identidad es objeto de disputa entre actores (Alegría 2004). En la misma línea de pensamiento, Criado Aguilar (2005) puntualiza que la nueva dinámica del patrimonio cultural tiene como explicación la emergencia de los valores locales, es decir, "la fuerza de lo singular, la importancia de lo diferente como sustantivo de la misma lógica global que conduce hacia culturas homogéneas” (53). Al hablar de lo local puede apreciarse que el PCI también tiene su espacialidad, ya que se manifiesta en lugares concretos y se resignifica por medio de la dinámica particular de diferentes grupos sociales. De ahí que García Canclini (1989) proponga reformular el patrimonio en términos de capital cultural y lo conciba como proceso social que se acumula, reconvierte y es apropiado en forma desigual por diversos sectores 
y que puede ser analizado como espacio de luchas - materiales y simbólicasentre los diferentes grupos (182).

Dentro de este marco más amplio, se inscribe el patrimonio cultural inmaterial como fuente de diversidad, identidad, creatividad y, además, práctica y conocimiento de quienes lo portan (Bouchenaki 2004; Kirshenblatt-Gimblett 2004). Su definición bajo este signo surge en el contexto de la Unesco y se conceptualiza e institucionaliza con la Convención del 2003. Si bien nace ligado a la necesidad de su protección, su enfoque se amplía hasta el punto de entender por patrimonio inmaterial "los usos, representaciones, expresiones, conocimientos y técnicas, junto con los instrumentos, objetos, artefactos y espacios culturales que le son inherentes, que las comunidades, los grupos, y en algunos casos los individuos reconozcan como parte integrante de su patrimonio cultural” (Unesco 2003, art. 2.1). Tal concepto puede hacerse presente en las tradiciones y expresiones orales, en las artes del espectáculo, en los usos sociales, rituales y actos festivos, en los conocimientos y usos relacionados con la naturaleza y el universo, así como en las técnicas artesanales tradicionales (art. 2.2). Uno de los aspectos más significativos de esta convención, el cual marcó un precedente en el ámbito internacional, es el rol central que se les da a las comunidades culturales asociadas al patrimonio inmaterial.

No obstante, consensuar esta definición no fue una tarea sencilla. El primer debate en las reuniones de especialistas convocados por la Unesco se dio a nivel de los desafíos epistemológicos de su enunciación. Se debió llegar a un acuerdo respecto del uso del término intangible (en inglés) por las dificultades que encontraron los expertos para adoptar otros posibles, tales como folclore, patrimonio oral, cultura tradicional, cultura expresiva, modo de vida, cultura etnográfica, costumbres, cultura popular o patrimonio cultural vivo. Además, como señala Sánchez Carretero,

A las dificultades epistemológicas de las definiciones de cada uno de estos términos, se unen las cargas ideológicas de los mismos, las particulares historias disciplinarias en cada país y las complicaciones para traducir conceptos, tanto por lo que denotan como también por lo que connotan. $(2005,149)$

En este sentido, se recuperó también el cuestionamiento del cual estaba siendo objeto el concepto antropológico de cultura como categoría analítica. Esta dejó de concebirse como un consenso unánime entre personas que conforman una comunidad y comenzó a percibirse "como un lugar de contestación”, cuya característica distintiva es que los pueblos están "recreándola continuamente” 
(Van Zanten 2004, 37). Es decir, tanto la cultura como el patrimonio fueron perdiendo su discrecionalidad previa, en términos de rasgos y poblaciones, para ser considerados como prácticas y procesos de producción de sentidos, como "praxis abierta y atravesada por relaciones de poder que pueden generar la naturalización de lo arbitrario así como la puja por hacer emerger significados alternativos” (Briones 1998, 6). El mismo concepto de cultura hizo que fuera redundante “el doblete material-inmaterial” del patrimonio (Sánchez Carretero 2005, 151), ya que ambas dimensiones son indivisibles (Bouchenaki 2004; Dawson 2004; Kurin 2004; Sánchez Carretero 2005; Van Zanten 2004). Las artesanías, por ejemplo, como lo expresa Kurin (2004), son parte de la cultura material, pero el conocimiento y las habilidades que hay que desarrollar para producirlas constituyen bienes intangibles, por lo cual una clasificación no se puede escindir de la otra. Por muchos años, el patrimonio cultural estuvo enfocado en la cultura material, de modo que el patrimonio inmaterial quedó relegado del debate. Una vez incorporado, fue necesario romper con las contradicciones que presentaba el binarismo implícito entre material e inmaterial ya que, en este contexto, tal binarismo “no podía sostenerse ontológicamente” (Sánchez Carretero 2005, 151).

Por otra parte, el concepto de patrimonio ha sido abordado desde la perspectiva de la economía política, que lo analiza como economía moral: "El patrimonio es un tipo de producción cultural que, en sus esfuerzos por proteger otras producciones culturales, ha usado de manera sostenida lógicas y modelos de aislamiento, oposición y valoración propios de la esfera de los intercambios económicos” (Montenegro 2010, 115). La necesidad de proteger ese patrimonio es vista como un imperativo moral con el propósito de evitar su uso inadecuado o su transformación radical. Montenegro $(2010,121)$ señala que los argumentos de defensa del patrimonio empiezan a pensarse más como “negociaciones”, debido a la escalada de las economías culturales que se dan de la mano del turismo cultural y de la institucionalización de la economía de intangibles, es decir, los derechos de autor, las patentes, el llamado know how, etc.

Precisamente esta cuestión está siendo objeto de debate, en los niveles tanto nacional como internacional, con relación a la necesidad de proteger los derechos colectivos de propiedad intelectual, una cuestión que es escasamente contemplada en el derecho internacional ${ }^{2}$. Otra cuestión interesante de mencionar es

2 En los últimos años, esta cuestión ha sido objeto de debate en organismos internacionales, como la Organización Mundial de la Propiedad Intelectual (OMPI) y la Unesco, entre otros, pero no ha sido posible aún llegar a un consenso sobre las figuras legales aplicables. Se han presentado varias propuestas en la Organización Mundial del Comercio (OMC) y en la OMPI, con vistas a reforzar las normativas internacionales sobre patentes para que se exijan formas específicas de divulgación con relación a los conocimientos tradicionales y los 
la tensión que puede generarse entre la protección de un patrimonio inmaterial que pretende ser entendido como común -y, por ende, libremente transmitido a las generaciones venideras-y los intereses económicos de sus portadores, que impulsan a estos a conservar el control sobre saberes, técnicas u oficios que, por su carácter único u original, tienen un especial reconocimiento en el mercado de las artesanías (e. g., el caso de la tejedora pampeana Ercilia Cestac en la provincia de Buenos Aires, analizado por Pedrotta et al. 2013). Todo ello, además, en un contexto en el cual la propiedad de los pueblos indígenas sobre su patrimonio cultural ha alcanzado el estatus de derecho humano y, por ende, los Estados están obligados a cumplir con los requisitos del consentimiento previo, libre e informado que impone el Convenio 169 de la OIT, norma ampliamente receptada por los sistemas normativos latinoamericanos (Endere 2014). No obstante, es necesario aclarar que los conocimientos que elaboran, mantienen y difunden los pueblos indígenas reconocidos como tales se consideran un cuerpo de conocimientos más precisos que los tradicionales, ya que estos últimos pueden pertenecer a otras comunidades locales y culturales que no están reconocidas como indígenas.

\section{La protección legal del patrimonio cultural inmaterial en Argentina}

Si la conceptualización del patrimonio cultural intangible genera nuevos desafíos, su salvaguarda mediante instrumentos legales y de gestión no es menos sencilla. En el caso de Argentina, la cuestión es particularmente compleja debido a las particularidades de su sistema legal, de tipo federal, y a la dificultad de

recursos genéticos o biológicos, pero no se ha adoptado aún ninguna norma vinculante. No obstante, existen normas más generales de derecho público internacional que brindan un marco jurídico y político general (e. g., el Convenio sobre la Diversidad Biológica, el Tratado Internacional de la FAO, las Disposiciones Tipo OMPI-Unesco sobre la Propiedad Intelectual de las Expresiones Culturales Tradicionales o del Folclore de 1982, las Directrices de Bonn sobre Acceso a los Recursos Genéticos y Participación Justa y Equitativa en los Beneficios Provenientes de su Utilización, la Convención de las Naciones Unidas de Lucha contra la Desertificación, la Declaración de las Naciones Unidas sobre los Derechos de los Pueblos Indígenas, etc.). Algunos países que cuentan con una gran diversidad biológica y cultural, donde viven comunidades que mantienen estilos de vida tradicionales, se han preocupado por proteger los conocimientos tradicionales. Por ejemplo, los países miembros de la Comunidad Andina adoptaron un Régimen Común sobre Acceso a Recursos Genéticos (Decisión 391). Esta cuestión no ha sido abordada por la legislación argentina (para una discusión más amplia sobre este tema, ver Endere y Mariano [2013]). 
actualizar la normativa vigente en los niveles nacional y provincial. Si bien este país ratificó en el 2006 la Convención de la Unesco mediante una ley del Congreso y, por ende, la incorporó a su sistema legal, en uso de los mecanismos democráticos que establece la Constitución Nacional, no ha habido en general un debate abierto respecto de los mecanismos de instrumentación de las políticas entre las diferentes instancias de gobierno que deben implementarlas ni con los actores sociales involucrados. Tampoco se cuenta con una ley federal de presupuestos mínimos del patrimonio cultural intangible a la cual deban adecuarse las normas provinciales (Mariano y Endere 2013). Solo algunas provincias regulan su PCI a través de normas especiales (e. g. Ciudad Autónoma de Buenos Aires y Mendoza), la mayoría de las cuales incluyen al PCI como un ítem dentro del patrimonio cultural, pero no contienen disposiciones específicas dedicadas a su salvaguarda. Otras provincias solo poseen leyes que protegen algunos aspectos del PCI, como las artesanías, las expresiones folclóricas, etc.

En el 2006, luego de haberse ratificado la Convención de la Unesco, la Secretaría de Cultura de la Nación designó a la Dirección Nacional de Patrimonio y Museos como el órgano encargado de llevar a cabo la salvaguarda del PCI en el territorio nacional. Esta dirección, a su vez, creó el Programa Nacional de Patrimonio Inmaterial, cuyo objetivo consiste en identificar, inventariar y registrar dichos bienes, incorporando un diseño y líneas generales de acción basados en la experiencia realizada en Brasil, que cuenta con un Registro de Bienes Culturales de Naturaleza Inmaterial desde el 2000 (para un análisis detallado del caso brasileño, ver Lima Filho, Ferreira y Abreu [2010]). Asimismo, dicha dirección tiene a su cargo la promoción y el desarrollo de las acciones de investigación, divulgación, valorización y salvaguardia del PCI en todo el territorio de la nación argentina, todo lo cual debe ser implementado de común acuerdo con las provincias. No obstante, aún no se ha avanzado en la definición de criterios de implementación.

A nivel provincial, se han producido algunos avances normativos. Un importante hito en la materia fue la Ley n. 1227 de Patrimonio Cultural que sancionó la Ciudad Autónoma de Buenos Aires en el 2003, así como la Ley n. 1535 del 2004, que creó el Atlas del Patrimonio Cultural Inmaterial de la Ciudad de Buenos Aires, y puso en marcha la investigación y el registro de fiestas, celebraciones y rituales que adquieren especial significación para la memoria, la identidad y la vida social.

Asimismo, la provincia de Mendoza posee la Ley de Patrimonio Cultural n. $6034 / 93$ (texto ordenado en el 2001) y ha sancionado el nuevo Decreto 
Reglamentario n. ${ }^{\circ} 1882 / 09$, que brinda un marco jurídico de protección a todo el acervo de bienes culturales, sean materiales o inmateriales.

Por su parte, la provincia de Buenos Aires, donde se encuentra el municipio de Olavarría, se caracteriza por la vetustez de su normativa patrimonial. Frente a esta situación, algunos municipios han mostrado interés en desarrollar sus propias políticas de protección.

Cabe aclarar que los mecanismos de participación ciudadana no están contemplados de manera explícita en la normativa patrimonial argentina, a diferencia de lo que sucede para cuestiones ambientales, respecto de las cuales la legislación exige la implementación de mecanismos de consulta a través de audiencias públicas. En el caso de bienes culturales que formen parte del patrimonio cultural indígena, la normativa vigente exige el consentimiento previo (Ley $n .^{\circ} 25517$ ), pero el procedimiento para su obtención aún no ha sido legalmente implementado.

\section{Caracterización del patrimonio inmaterial olavarriense}

En este trabajo se toman como caso de estudio las manifestaciones más evidentes del PCI del municipio de Olavarría, que surgieron a partir de una caracterización general pero no exhaustiva. El partido de Olavarría ${ }^{3}$ posee una superficie de 7.715 $\mathrm{km}^{2}$. Allí confluyen una gran diversidad de expresiones culturales y costumbres propias de grupos con diferentes orígenes que fueron construyendo su etnicidad de manera diversa. Desde finales del siglo XIX y hasta mediados del siglo XX, Olavarría se convirtió en el destino de numerosas corrientes migratorias. Alemanes, franceses, portugueses, vascos, italianos, españoles, sirios y libaneses, entre otros, se sumaron a los habitantes locales y formaron una población intercultural. A mediados del siglo XX, floreció la inmigración de origen latinoamericano, dentro de la cual fue la boliviana la más numerosa. Actualmente, la comunidad de Olavarría está conformada por descendientes de grupos indígenas (aunque no legalmente organizados como comunidades indígenas), los autodenominados “criollos”, e inmigrantes que se radicaron en el partido en los últimos 150 años.

\footnotetext{
3 En Argentina, la unidad territorial que corresponde a la entidad política de municipio se denomina partido y comprende áreas rurales y urbanas, incluidas la ciudad cabecera y varias localidades menores.
} 
Como consecuencia, existe una gran variedad de manifestaciones y celebraciones vinculadas con diversas tradiciones culturales, susceptibles de ser salvaguardadas, desde la perspectiva del patrimonio inmaterial, por el gobierno local. Cabe destacar que este municipio no se caracteriza por ser un distrito turístico y los participantes pertenecen, en su gran mayoría, a la población local.

Desde finales del siglo XIX, por ejemplo, se asentaron en la ciudad los descendientes de alemanes del Volga. Esta denominación proviene de 1763, cuando la emperatriz Catalina II de Rusia, con el propósito de incentivar la inmigración, prometió a un grupo de alemanes concederles tierras a orillas del río Volga y mantenerles ciertos privilegios, como el idioma, la profesión y la religión. Allí vivieron dedicados a realizar trabajos agrícolas y lograron, a su vez, un alto crecimiento demográfico y económico. Disconformes con su situación, un siglo más tarde comenzaron a emigrar a diferentes países, entre ellos Argentina (Duguine 2010). Sus descendientes aún mantienen su forma original de organización social en las colonias del partido (e. g. colonias Hinojo, San Miguel y Nievas). En ellas todavía es posible observar el particular diseño de sus plantas urbanas, así como ejemplos de la arquitectura tradicional y la presencia de actores sociales que promueven, desde la práctica, la enseñanza de la lengua, así como la transmisión de costumbres, saberes, relatos y cuentos. Es tradicional la Fiesta de la Kerb ${ }^{4}$, una celebración que conmemora el aniversario de su fundación y durante la cual se realizan desfiles, danzas, espectáculos musicales y venta de comidas típicas y productos artesanales. Estas actividades son organizadas por la propia comunidad, aunque cuentan con apoyo del municipio. La visita a las colonias y la degustación de su gastronomía forman parte del repertorio turístico tradicional de Olavarría, aunque se trata de actividades de escala regional.

En 1998 se inauguró un museo municipal en la colonia Hinojo, gracias a la iniciativa del museólogo Ariel Edgardo Chierico y al apoyo de un grupo de vecinos de la localidad. Este museo se complementó luego con otro, también municipal, creado en el 2000 en la colonia San Miguel, por iniciativa de la familia Stoessel. La misión de estos museos es "albergar y custodiar" la memoria de los alemanes del Volga que se instalaron en esta región a fines del siglo XIX. Ambos museos se emplazan en antiguas viviendas familiares de arquitectura tradicional alemana. Cuentan con exposiciones permanentes donde se pueden conocer las historias de estos inmigrantes y las peripecias que tuvieron que sortear desde su partida del sur de Alemania, la vida a orillas del Volga, el viaje a través del

4 La Fiesta de la Kerb es tradicional en las colonias de alemanes del Volga de Argentina y en ella se celebra el día del santo patrono de cada comunidad, que coincide con el aniversario de su fundación. 
Atlántico y su instalación definitiva en el centro bonaerense. También presentan los resultados de los estudios sobre la genealogía de las familias más tradicionales, relatos de vida y costumbres propias del siglo XIX, a la vez que se exhiben numerosos objetos donados por los descendientes de los primeros habitantes de las colonias.

Todos estos aspectos son significativos, ya que aluden a actores sociales que resignifican su cultura, su etnicidad y van generando, en su vida cotidiana, un espacio en el cual vivir lo colectivo-comunitario. De cierto modo, todas las representaciones en juego permitirían un análisis amplio en términos de patrimonio cultural, y uno más específico, en términos de patrimonio arquitectónico e histórico. Desde la perspectiva de lo inmaterial, este escenario haría posible construir iniciativas de gestión en las que se apunte a "coproducir con los otros, considerando los diversos contextos de intercambios, relaciones y conflictos" (Lacarrieu y Álvarez 2008, 23).

Otro ejemplo son los grupos de inmigrantes que conforman la Asociación de Residentes Bolivianos en Olavarría, que comenzaron a llegar a partir de la década de los cincuenta, atraídos por la promesa de trabajo y prosperidad que la ciudad ofrecía. El despliegue de danzas y manifestaciones que esta comunidad realiza en sus diferentes celebraciones hizo que los "bolivianos" en Olavarría fueran configurándose como actores sociales visibles, con la posibilidad de comunicar sus representaciones identitarias por medio de acciones concretas. En la actualidad, cada fin de semana próximo al 6 de agosto, las familias se reúnen para celebrar la fiesta patronal. Dos barrios se unen y concurren, además, vecinos olavarrienses. De esta forma, entre las calles de los barrios Villa Mailín y Provincias Unidas, los festejos por la independencia (6 de agosto) se articulan con la veneración a la Virgen de Copacabana (5 de agosto), en lo que constituye una festividad patria y religiosa a la vez. Por sus características, "la fiesta impacta a todos los sentidos" (Giorgis 2004, 33), por medio de los gestos, los adornos llamativos, la música, las danzas, los puestos de comida y bebida, los trajes coloridos de los bailarines y las banderas, etc. Durante estos dos días pueden identificarse algunos elementos propios de las prácticas católicas, como el rezo de la novena a la Virgen, la celebración de misas, procesiones y ritos individuales, sumados a prácticas tradicionales que se incorporan en este festejo, como las danzas o el intercambio de comida y bebida entre los participantes. Leído en términos de patrimonio inmaterial, este escenario pone de relieve tradiciones y expresiones orales, así como usos sociales, rituales y actos festivos susceptibles de ser valorados como significativos para la comunidad (Unesco 2003, art. 2.2). 
Otro caso a considerar es la Fiesta del Inmigrante, organizada en el mes de septiembre por la municipalidad de Olavarría, que desde hace dos décadas forma parte de la agenda cultural municipal. El evento congrega a los integrantes de las colectividades más representadas localmente - portuguesa, española, francesa, italiana, alemana, suiza, libanesa, boliviana y vasca, entre otras-. En la mayoría de los eventos pueden identificarse elementos típicos de cada cultura, como la gastronomía, la vestimenta y las danzas.

Un ejemplo diferente es el Festival de Doma y Folclore, en el que se pone en juego el repertorio patrimonial criollo, vinculado a las tradiciones del gaucho y del ámbito rural. Este festival se realiza desde hace diez años y su impacto en importancia y cantidad de público fue aumentando al punto de convertirse en el Festival Nacional de Doma y Folclore de la provincia de Buenos Aires. A lo largo de tres días se llevan a cabo jineteadas ${ }^{5}$, certámenes de payadas $^{6}$ y peñas ${ }^{7}$, y son invitadas figuras destacadas del folclore nacional. Si bien el costo de la entrada es oneroso, no solo concurre un amplio abanico generacional y social de habitantes de la ciudad y, sobre todo, del campo, sino también personas de otras ciudades y provincias.

Para referirse a la diversidad de manifestaciones culturales que componen el PCI de Olavarría y los actores sociales involucrados, es significativo mencionar que incluso existen grupos de descendientes de pueblos originarios. Uno de ellos está constituido por la comunidad mapuche Pillan Manke, que organiza durante el mes de agosto una celebración ancestral para honrar a la Madre Tierra y el ciclo de la naturaleza. Se realizan ofrendas acompañadas de cánticos en una ceremonia de carácter público que tiene lugar en el parque zoológico municipal La Máxima, donde se crían -y se liberan periódicamente-cóndores, aves consideradas sagradas por los pueblos originarios.

Por otra parte, la Escuela Municipal de Orfebrería y Artesanías Tradicionales también puede ser analizada como un ámbito de transmisión de usos, conocimientos y técnicas artesanales tradicionales. Esta escuela se creó en 1978 y tiene como antecedente los saberes de artistas europeos que se radicaron en la ciudad a fines del siglo XIX. Con el tiempo, se consolidó el carácter distintivo de la orfebrería olavarriense, que consiste en una amalgama de estilos entre

5 La jineteada gaucha es un deporte ecuestre característico de Argentina y el Cono Sur, que consiste en que un jinete debe sostenerse varios segundos sobre un caballo no domesticado.

6 La payada es un arte poético musical perteneciente a la cultura hispánica que adquirió un gran desarrollo en Argentina. En ella, el payador improvisa un recitado en rima acompañándose de una guitarra.

7 La peña es una agrupación cultural argentina en cuyas reuniones se suceden eventos musicales con diversos grupos de canto y danza y se ofrecen comidas típicas. 
lo criollo y lo europeo. Para finales de los setenta, Olavarría ya era considerada pionera en la institucionalización de la orfebrería a nivel nacional (Conforti, Mariano y Endere 2009). En este contexto, la creación de la escuela contribuyó a fortalecer y popularizar aquella, al ofrecer un espacio de educación no formal en el cual los maestros artesanos enseñan a los aprendices trabajando con ellos las piezas. De algún modo, la escuela ayudó a mantener la continuidad de esta tradición gracias a la transmisión de conocimientos que se han ido resignificando con el tiempo, entre las diferentes generaciones.

Por último, y para ampliar el repertorio de manifestaciones susceptibles de ser analizadas desde la perspectiva inmaterial, cabe mencionar otro evento que en los últimos años ha crecido en convocatoria y trascendencia, hasta convertirse en una de las fiestas más populares de la ciudad: el carnaval. Desde el gobierno municipal se difunde la organización del denominado Corso Oficial, que se extiende en fechas variables desde mediados de enero hasta mediados de febrero de cada año y que, a lo largo de su historia, fue ocupando diferentes escenarios. Dada la creciente participación ciudadana, el Corso se fue trasladando a lugares más amplios que permitieran el despliegue de murgas, grupos de percusión, batucadas y otros protagonistas que escoltan al Rey Momo ${ }^{8}$, así como ubicar sillas para el público y puestos de venta. La necesidad de contar con un espacio propio y adecuado ha movido a las autoridades a construir un "corsódromo" permanente. En esta manifestación confluyen viejas y nuevas tradiciones, algunas locales y otras traídas desde lejos, como las danzas bolivianas.

Estas son las expresiones más relevantes del patrimonio inmaterial local que se pudieron observar en un diagnóstico inicial. Sin embargo, es necesario contar con información más precisa que contribuya a comprenderlas como parte de un patrimonio que se desea salvaguardar. Y se entiende por salvaguarda todas aquellas "medidas encaminadas a garantizar la viabilidad del PCI”, incluidas "la identificación, documentación, investigación, preservación, protección, promoción, valorización, transmisión y revitalización de este patrimonio en sus distintos aspectos" (Unesco 2003, art. 2.3). Asimismo, es importante develar las contradicciones que puedan generar las activaciones patrimoniales y tratar de evitar sus efectos negativos. Por ello, para poder caracterizar mejor parte del PCI olavarriense y dar cuenta de su multiplicidad, se eligió como estrategia identificar diversos actores sociales y revelar las manifestaciones que producen. Este recorte ayuda no solo a comprender la dimensión social de las prácticas y su relación con la identidad local, sino también a pensar en los desafíos y conflictos que 
la misma gestión del patrimonio implica. El caso de las prácticas y representaciones culturales bolivianas es particularmente interesante porque su valoración desde el punto de vista del PCI, lejos de asociarse a una lógica de fortalecimiento del mercado a través del turismo cultural de una expresión ya consolidada y socialmente aceptada — como podría ser el caso de algunas manifestaciones culturales europeas-, contribuiría a visibilizar un grupo excluido por razones de clase y de nacionalidad, y a promover su inserción social a través del reconocimiento de su aporte a la diversidad cultural local.

\section{Herramientas metodológicas para la gestión del PCI local}

Como se señaló anteriormente, uno de los objetivos del equipo de investigación Patrimonia es crear un sistema de información que reúna datos relativos a las expresiones y manifestaciones que componen el PCI del centro de la provincia de Buenos Aires, tomando como una primera instancia el municipio de Olavarría. Asimismo, se busca generar políticas de patrimonio no subordinadas a intereses externos, sino llevadas a cabo "de cara a la gente" (Belli y Slavutski 2005, 15) y "en función de la importancia que se [le] asigna [al patrimonio] en la memoria colectiva y en la integración y continuidad de la cultura presente” (Bonfil Batalla 1991, 130). Es por ello que no se trata de efectuar un registro en libros (como en Brasil), ni en un atlas (como en la ciudad de Buenos Aires) ni tampoco en inventarios en el sentido tradicional del término, sino de generar un instrumento concreto de gestión que agilice la disponibilidad de la información y posibilite la toma de decisiones. En este sentido, se propone poner la gestión del patrimonio cultural en contexto, es decir, tener presentes cuestiones centrales como las relaciones sociales y simbólicas que encierran las manifestaciones, así como la diversidad de visiones, sentidos, tensiones y conflictos que producen.

Así, se considera una cuestión crucial la participación de los actores sociales en los procesos de identificación y en la elaboración de diferentes estrategias y políticas tendientes a su manejo. En este contexto, se introduce la idea de una gestión participativa como punto de partida para la efectividad de cualquier acción destinada a pensar y promocionar el reconocimiento y la visibilidad de la diversidad cultural. Tal como lo ve la antropología, el desafío actual consiste en contribuir al fortalecimiento de estos procesos de gestión a través de la investigación, 
para instalar y poner en escena el tema del PCI en un partido como Olavarría, que se caracteriza por tener una población diversa, pero que carece de políticas favorables a sus múltiples expresiones culturales. Esto implicaría promover, en términos de Rotman (2004), un proceso intencional con un doble movimiento: de identificación y valorización dentro de las agrupaciones, por un lado, y de reconocimiento por parte de la sociedad y las agencias estatales, por el otro.

En Olavarría el actual gobierno ha desarrollado la metodología de mesas de diálogo y consenso como modo de negociar, con los diferentes grupos de interés involucrados, las diversas temáticas y etapas de la planificación y el ordenamiento territorial del partido. La inclusión de los componentes materiales e inmateriales del patrimonio cultural local en este proceso constituye una doble oportunidad: por un lado, para promover el debate en ámbitos que suelen contar con amplia convocatoria y participación; por otro, para lograr que estas cuestiones sean tenidas en cuenta en las futuras políticas territoriales y de uso del suelo. En el caso del PCI, los actores involucrados incluyen necesariamente a comunidades, grupos e individuos portadores, quienes a través de ese mecanismo prestarán (o no) su consentimiento para la inclusión de sus manifestaciones culturales, así como de las modalidades de salvaguarda, en el marco de un proceso participativo de gestión que prevé instancias periódicas de reunión, discusión y revisión de políticas.

El objetivo final de la inclusión del PCI no es necesariamente patrimonializar (en los términos burocráticos de una declaratoria), sino revelar aquellas expresiones del PCI que son apropiadas y resignificadas por las diferentes generaciones a lo largo del tiempo para contribuir, mediante una gestión participativa, a su visibilización y valoración social. En este sentido, se espera generar un producto con una clara aplicabilidad social, que incluya a los grupos de interés y al PCI en políticas concretas de fortalecimiento de prácticas culturales olavarrienses.

El desarrollo de la tecnología informática ha hecho posible gestionar y analizar la información de manera muy sencilla. Esto es posible gracias al avance en el manejo de modernas herramientas que permiten generar modelos informatizados del mundo real, con sistemas de referencia ligados al espacio. Los sistemas de información geográfica (SIG) son sistemas de hardware y software y procedimientos diseñados para facilitar la obtención, manipulación, análisis, modelación y salida de datos espacialmente referenciados, que resultan de utilidad para resolver problemas complejos de planificación y gestión (Ladrón de Guevara 2011, 35; NCGIA 1990). El éxito y la eficacia de un SIG se miden por el tipo, la calidad y la vigencia de la información con la que opera. Estos sistemas se pueden aplicar a aquellas actividades humanas en las que la información espacial tenga un papel determinante y que estén relacionadas con la planificación. 
Como ya se mencionó previamente, se busca adoptar un enfoque teórico y metodológico adecuado sobre el cual basar la práctica de la gestión del patrimonio inmaterial. En este caso, se propone utilizar el SIG como herramienta para identificar, registrar y reconocer institucionalmente al PCI para, de este modo, contribuir en la construcción de políticas de manejo efectivas, que garanticen su salvaguarda, visibilización y sostenibilidad.

La primera actividad consiste en crear un instrumento básico de compilación de la información. Se propone para ello elaborar una ficha que contenga todas las variables de interés. La planilla de compilación de datos incluye variables generales denominadas categorías, de las cuales se desprenden otras más específicas que no son taxativas, sino que pueden ampliarse en el futuro, de ser necesario. La selección de las variables utilizadas en la ficha es el resultado de observaciones participantes efectuadas en los diversos eventos descriptos en el apartado "Caracterización del patrimonio inmaterial olavarriense".

La entidad inmaterial madre se denomina manifestación cultural inmaterial y con ella se alude a cada estrategia por medio de la cual un grupo o individuo se torna visible y manifiesto para los demás grupos y para sí mismo (Giménez 2005). De ella se desprenden las siguientes categorías: identidad, participación, acceso, periodicidad, coordenadas, carácter, valoración, actividades, protección legal, diseño expresivo, transmisión y elementos simbólicos (figura 1).

Figura 1. Entidad madre y categorías más generales para clasificar el PCI de Olavarría

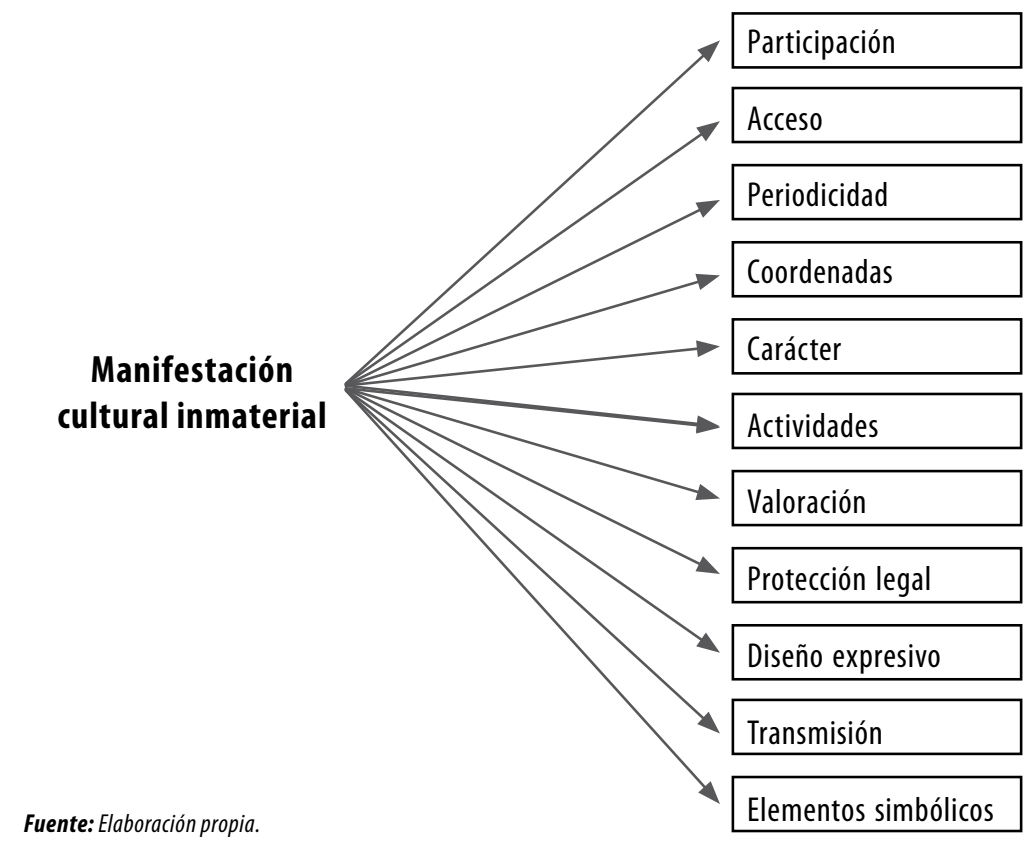


Algunas de estas categorías pueden desglosarse en variables que refieren a cuestiones más sencillas de catalogar —como participación, acceso, periodicidad y coordenadas - que, a su vez, implican variables más taxativas —como individual o colectiva para participación, o bien pública o cerrada para acceso(figura 2).

Figura 2. Ejemplo de categorías y variables de definición más "objetiva"

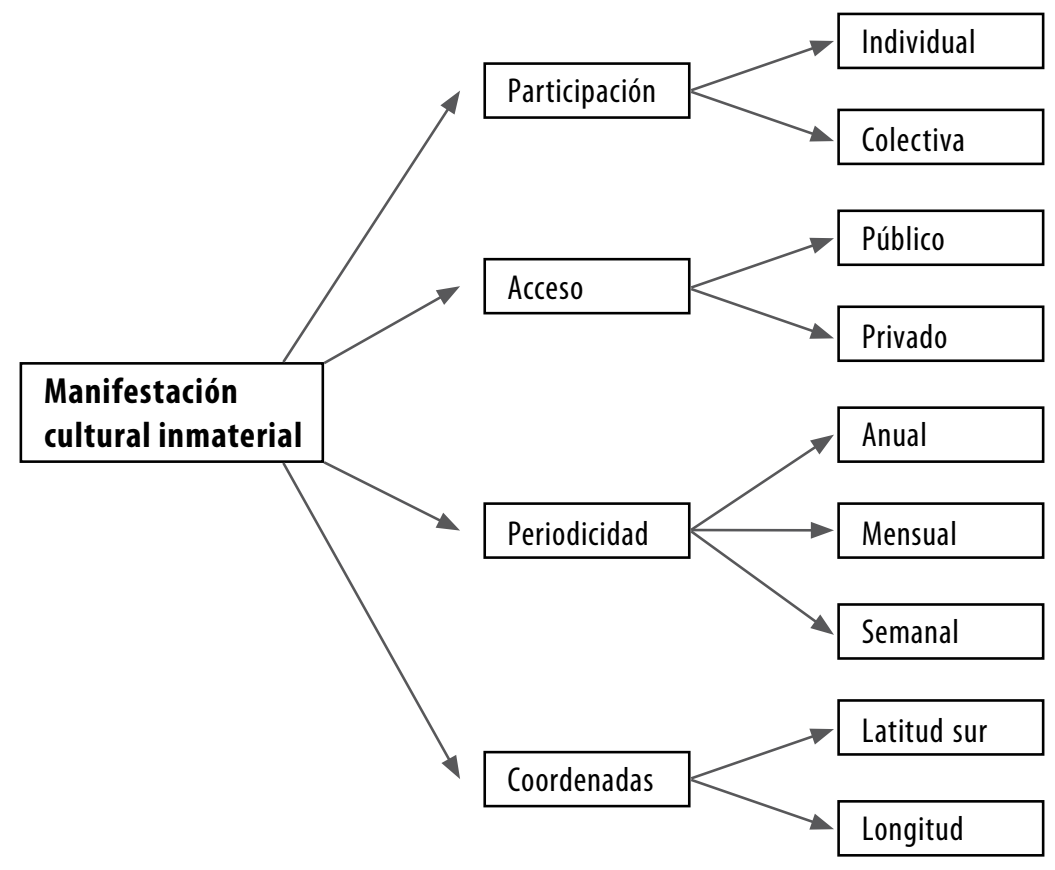

Fuente: Elaboración propia.

Otras categorías, por el contrario, necesitan definirse porque involucran diferentes procesos de identificación social (tales como valoración, identidad, diseño expresivo, etc.). Su utilización puede parecer ambigua y, por ello, es necesario definir cada una de ellas y las variables que la componen.

A través de la variable identidad, se buscó catalogar a los individuos, grupos y comunidades participantes en relación con su adscripción étnica/cultural. En cuanto a la información revelada, se establecieron tres variables derivadas: la identidad inmigrante, que refiere tanto a la generación de inmigrantes como a sus descendientes y a aquellos que se adscriben étnicamente como tales; la identidad indígena, que reúne a las diferentes etnias que se reconocen como descendientes de pueblos originarios, y, por último, la identidad criolla que, en 
contraste con las otras dos, remite al conjunto de personas que no se identifican como inmigrantes ni como indígenas y que se vinculan con las tradiciones patrias o el mundo rural. Esta división introduce la posibilidad de individuos que se consideren, al mismo tiempo, inmigrantes e indígenas. Sin embargo, estas variables siguen siendo muy generales, ya que no especifican el país de origen ni tampoco la adscripción a un grupo étnico particular. Por ello, tanto la identidad inmigrante como la indígena están acompañadas por listas de control que complementan la información necesaria para referirse a la identificación social. Cada una de estas listas tiene una denominación. Por ejemplo, país de origen para la identidad inmigrante, la cual contiene la totalidad de las nacionalidades identificadas hasta el momento en el partido de Olavarría.

Podría incluirse, de ser necesario, una cuarta variable, denominada otra, para cuando no se trate de una identificación de tipo étnico. Puede tratarse de una manifestación vinculada con el mundo del trabajo - e. g., décadas atrás se celebraba en Olavarría la Fiesta de la Seguridad o la Fiesta del Cemento en las villas obreras industriales de Sierras Bayas y Loma Negra, respectivamente-.

Otra categoría amplia es la de carácter, por la cual se clasifican las manifestaciones en razón de sus rasgos distintivos. En este caso, se decidió subdividirlas en carácter religioso, carácter tradicional/patrio y carácter popular. En una manifestación de tipo religioso, se pone en juego un conjunto variado de fenómenos, prácticas y creencias que engloban tanto la mitología como la fe; los credos, los ritos, las oraciones e, incluso, las instituciones involucradas. En un evento de tipo tradicional/patrio, si bien pueden existir algunas actividades de carácter religioso, el sentido principal se relaciona con las tradiciones propias del gaucho argentino, así como con las vinculadas a la historia nacional y los símbolos patrios. Bajo el carácter popular se buscó agrupar, por su parte, los diferentes eventos que convocan a un número significativo de asistentes.

Ahora bien, hay un conjunto de elementos que podrían ser analizados como entidades culturales inmateriales en sí mismas. Es decir, podrían ser entendidos como un todo y desglosados a partir de múltiples variables. Se trata de los grupos de danzas, la gastronomía, las artesanías y la música. Sin embargo, aquí serán analizados como variables que responden a la categoría mayor denominada diseño expresivo, la cual se subdivide, a su vez, en diseño expresivo manual, visual, sonoro y gestual (figura 3).

Cada una de estas últimas variables se eligió para prácticas como bailes, comparsas, trajes típicos (visual); murgas, batucadas, cantos, narraciones literarias (sonoro); artesanías, ornamentos, comidas típicas (manual), y teatralizaciones (gestual). 
Figura 3. Variable diseño expresivo y algunos de sus componentes

\section{Manifestación cultural inmaterial}

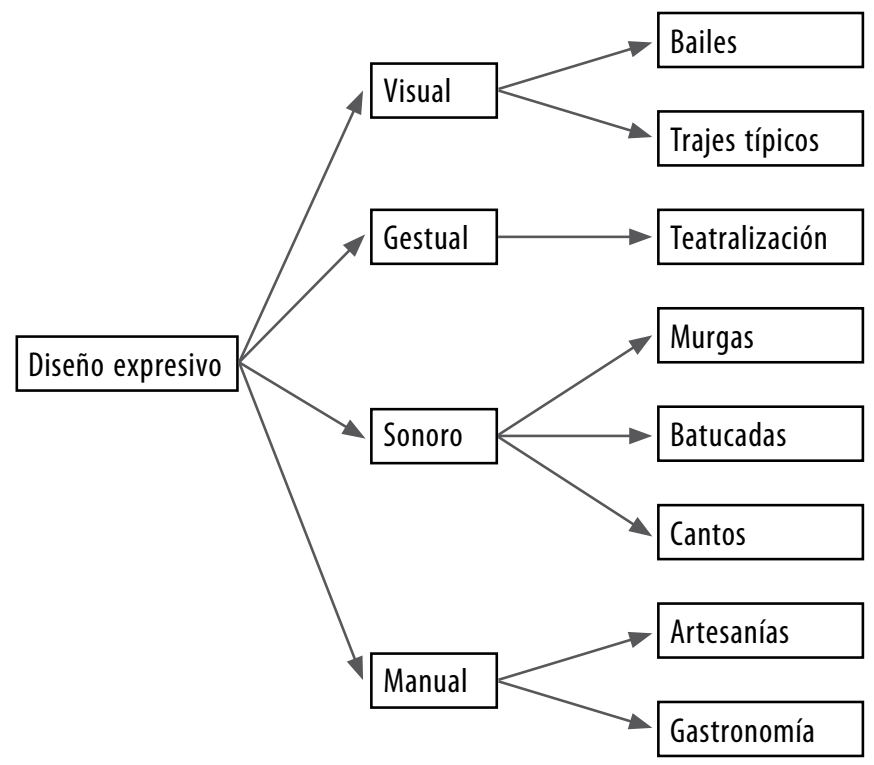

Fuente: Elaboración propia.

Así como la categoría transmisión se reduce a dos variables - oral y escrita-, la categoría actividad implica un conjunto de prácticas de lo más diversas, como por ejemplo las de carácter religioso - misas, procesiones, rezos, entierros, ofrendas, cantos-, pero también incluye acciones relacionadas con eventos de carácter patrio/tradicional, como jineteadas, juegos propios del campo, payadas, desfiles cívicos y militares, actos protocolares y conmemorativos de efemérides patrias y pialadas ${ }^{9}$, entre otras.

Valoración es una de las categorías más complejas de definir. Remite a las diferentes significaciones que les atribuyen los distintos actores sociales a las manifestaciones culturales inmateriales. Las variables definidas para esta categoría son: científica, para referirse a todo tipo de valoración de carácter experto, es decir, efectuada por especialistas de diferentes disciplinas; administrativa, para aquellas prácticas que implican la implementación de políticas y programas de gestión; política, para aludir a las valoraciones que realizan las autoridades de los diferentes niveles de gobierno; social/comunitaria, vinculada con la significación que le atribuye la población a nivel local, regional o nacional, y étnica, para referir

9 La pialada es un juego que consiste en enlazar las patas de un animal, generalmente caballos o vacunos. 
a las valoraciones de carácter más particular, vinculadas a un grupo específico. Finalmente, se incluyen dos variables más, las valoraciones turística y educativa, que poseen las manifestaciones inmateriales, ya sea por su atractivo para los visitantes, en el primer caso, o por su potencial didáctico y pedagógico, en el segundo. La valoración del turismo cultural como positivo es una característica común en la comunidad local, en especial en el caso de las colonias de alemanes del Volga, que ven en aquel una salida para superar el estancamiento económico y la pérdida gradual de población.

Por último, y para dar cuenta de una serie de elementos que aparecen recurrentemente en las diversas expresiones, se creó la categoría elementos simbólicos. Si bien se trata de una denominación en la que puede entrar un amplio espectro de elementos, con ella se alude, por ejemplo, a la presencia de emblemas, banderas, estandartes u objetos que tienen una importante carga simbólica en el marco de la manifestación cultural inmaterial de que se trate.

Respecto del modo de volcar los datos en el instrumento de gestión seleccionado, cabe señalar que se utilizan los números 1 o 0 para codificar la presencia (1) o ausencia (0) de la variable que se está evaluando. Por ejemplo, la celebración en honor de la Virgen de Copacabana que realiza anualmente la comunidad boliviana de Olavarría llevará un 1 en el campo de la variable carácter religioso y un 0 en el campo de la variable privada o cerrada al público. Esta numeración facilita el tratamiento cuantitativo de los datos. También puede haber codificaciones no binarias, que se utilizan para aquellos datos que pueden poseer tres o más valores. Una instancia de este tipo de codificación es la categoría protección legal, en la cual, por ejemplo, el 0 representa que la entidad no posee protección legal específica; el 1, que posee protección legal a nivel municipal; el 2, que tiene protección a nivel provincial, y así sucesivamente hasta el 5, que indicaría que la entidad patrimonial intangible goza de una declaratoria de la Unesco para su protección. En casos como este, se observa la necesidad de utilizar un criterio clasificatorio que considere la variabilidad y la jerarquía que existe entre las diferentes opciones, ya que, siguiendo el ejemplo inmediatamente anterior, no es lo mismo que la manifestación cuente con una declaratoria de interés municipal, que con una nacional o mundial.

Cuando los datos organizados de este modo se ingresan al sistema de información (SIG), este es capaz de realizar una selección, por ejemplo, agrupando solamente las variables cuyos campos se completaron con un número distinto de 0 . De este modo, el instrumento de gestión muestra exclusivamente las características de la entidad patrimonial por la cual se tiene interés en el momento de la consulta. Además, el sistema es capaz de ubicar los datos en el espacio geográfico, 
cosa que diferencia a los SIG de cualquier otra herramienta de almacenamiento y gestión de la información.

Por tratarse de un primer acercamiento metodológico hacia la difícil tarea de gestionar el PCI, este instrumento experimental deberá ser evaluado - y, posiblemente, reestructurado varias veces conforme pase el tiempo-. No obstante, podría constituir un modo útil y original de garantizar que toda la información esté disponible y en un solo lugar a la hora de ser requerida. Como propuso Laidlaw (1989): "Todo plan de manejo efectivo del patrimonio requiere tanto de la incorporación de estrategias como de un método eficiente de compilación de datos” (234).

La información volcada en cada una de las categorías y variables en las que se desglosan las manifestaciones seleccionadas no refiere todo lo que ocurre durante el desarrollo de estas, tal como lo haría un trabajo de tipo etnográfico a través de una descripción densa. No obstante, el SIG permite incluir, a través de vínculos, toda la información complementaria que exista sobre cada una de las manifestaciones analizadas (e. g., estudios previos, registros fotográficos, publicaciones, actuaciones administrativas, normativa específica, etc.).

\section{Discusión y conclusiones}

Cabe señalar que, hasta ahora, las propuestas de sistematización y valoración del patrimonio cultural en Olavarría han surgido desde los centros académicos, por ausencia de una gestión estatal, y con el objetivo de estudiar y problematizar los fenómenos que surgen en relación con aquel. De hecho, no existen políticas efectivas de gestión a nivel local ni provincial dirigidas a la preservación, valorización o visibilización del patrimonio material, y menos aún del PCI. Por ello, y teniendo como ejemplo experiencias previas de inventarios de PCI en otras regiones del país y del exterior, se comenzaron a desarrollar herramientas para implementar políticas de gestión dirigidas a salvaguardar el PCI del partido de Olavarría, y ya existe un preacuerdo con el municipio para su aplicación.

Es necesario puntualizar que la utilización del SIG para el PCI implica asumir un desafío importante, no solo porque existen muy escasos antecedentes nacionales al respecto, sino también por la dificultad que conlleva la construcción de categorías y codificaciones que involucren la diversidad de actores y actividades complejas y dinámicas. 
Se ha criticado a los inventarios por ser instrumentos estáticos, pasivos e impositivos, que dejan de lado los contextos vivenciales que envuelven y dan sentido a lo intangible, y porque excluyen la diversidad de matices, conflictos y contradicciones inherentes a tales contextos, con lo cual exponen las expresiones aisladas de las sociedades que las crearon y le dan un lugar excluyente a la opinión de los expertos. En respuesta a este argumento, se ha decidido someter la denominación y definición de las variables más críticas de la base de datos a la consideración de los diferentes actores sociales que llevan a cabo cada manifestación del PCI. En Colombia, por ejemplo, propusieron dejar de lado las fichas de inventarios y avanzar en el uso de metodologías cualitativas de investigación: la etnografía, por ejemplo, entendida como el puente de comunicación entre la identificación, la salvaguarda y el patrimonio (Santoyo 2006). El problema con este tipo de proceder es la falta de eficiencia en la obtención, el uso y el manejo de la información relevante e imprescindible a la hora de planificar estrategias de gestión concretas. Esto no quiere decir que se esté considerando irrelevante la etnografía, todo lo contrario. Lo que se propone es garantizar, en la práctica, el manejo y la salvaguarda de los bienes culturales inmateriales, y generar una herramienta para clasificar y jerarquizar la información, de modo que pueda ser usada de manera selectiva, de acuerdo a la finalidad de la tarea a realizar.

En el proceso de selección de las categorías, se buscó, sobre todo, evitar el error de objetivar y cosificar prácticas, o bien, de simplificar la misma diversidad. La Unesco (2003) propone definiciones amplias y flexibles que, utilizadas en un contexto local, suelen resultar inabarcables metodológicamente. Como lo expresa Lacarrieu (2010), representaciones, saberes y conocimientos son asuntos de la estructura simbólica que atraviesa toda la vida social. Sin embargo, como categorías resultan escasamente materializables de cara a los fines de la patrimonialización. Es decir, una de las principales cuestiones a resolver es la de la construcción de categorías que puedan referir los cambios e impidan el "congelamiento" de la dinámica propia de la realidad que se registra, al tiempo que reconozcan el contexto sociocultural de observación. Es en este contexto que se considera de gran importancia la etnografía para repensar las categorías y los sistemas de información. De hecho, a través de esta aproximación cualitativa es posible incorporar los propios saberes de los protagonistas y las conductas observables. El empleo del enfoque etnográfico (Guber 2011; Van Maanen 1995) y el uso de la perspectiva del actor permiten analizar la diversidad y la singularidad, y rescatar la lógica de la producción material y simbólica de los sujetos. En suma, el trabajo de campo antropológico no solo contribuye a identificar los grupos de interés y tomar contacto con distintas organizaciones intermedias (formales y 
no formales), sino que, además, posibilita promover instancias de diálogo en las cuales se reflejen los intereses, preocupaciones y demandas, y se den a conocer las redes de relaciones, los modos de participación y las formas de producción cultural de cada grupo.

Por todo ello, se escogió una herramienta para interrelacionar un amplio número de variables, una carga ilimitada de información adicional y cambios periódicos. Si bien se realizó un testeo inicial de la utilización del SIG aplicándolo a las festividades de la comunidad boliviana en Olavarría, la tarea futura consiste en completar los datos correspondientes al resto de las manifestaciones seleccionadas y cotejar las categorías empleadas con los diferentes actores sociales a través de entrevistas. A partir de los resultados que se obtengan, se introducirán las modificaciones necesarias.

En suma, elaborar y aplicar herramientas para la gestión del patrimonio inmaterial en Olavarría implica asumir el desafío de sistematizar una diversidad de manifestaciones con la participación de los actores sociales involucrados y poner en escena el tema del PCI en una región donde aún no existen políticas de manejo vinculadas con este. En esta tarea es imposible soslayar las dificultades, contradicciones y conflictos que implica la patrimonialización de las expresiones culturales del PCI (Chaves, Montenegro y Zambrano 2010). La participación, el diálogo, la negociación y la búsqueda permanente de consensos con los portadores de ese patrimonio son imprescindibles para generar mecanismos de gestión social y culturalmente sostenibles.

\section{Agradecimientos}

Este trabajo fue realizado en el marco de las investigaciones desarrolladas por Patrimonia (Estudios Interdisciplinarios de Patrimonio), Incuapa (Unidad Ejecutora Conicet-Unicen). Fue financiado con fondos provenientes de los proyectos PICT 0561/11, de la ANPCyT, y PIP 429/12, de Conicet, dirigidos por la Dra. María Luz Endere. 


\section{Referencias}

Alegría, Luis. 2004. "Museos y campo cultural: patrimonio indígena en el Museo de Etnología y Antropología de Chile". Revista del Centro Nacional de Conservación y Restauración 8: 57-70.

Belli, Elena y Ricardo Slavutski. 2005.

"Patrimonio: territorio, objetos, símbolos, personas. ¿Cuál es la disputa?”. Mundo de Antes 4: 13-22.

Bonfil Batalla, Guillermo. 1991. Pensar nuestra cultura. México D. F.: Alianza.

Bouchenaki, Mounir. 2004. Editorial de Museum Internacional. Intangible Heritage 221/222: 7-12.

Briones, Claudia. 1998. “(Meta)cultura del Estado-nación y estado de la (meta) cultura: repensando las identidades indígenas y antropológicas en tiempos de posestatalidad”. Brasilia: Serie Antropológica, Departamento de Antropología.

\section{Chaves, Margarita, Mauricio Montenegro} y Marta Zambrano. 2010. "Mercado, consumo y patrimonialización cultural”. Revista Colombiana de Antropología 46 (1): 7-26.

\section{Conforti, María Eugenia, Mercedes} Mariano y María Luz Endere. 2009. "El cincelado del orfebre: patrimonio cultural inmaterial de Olavarría. El caso de la Escuela Municipal de Orfebrería y Artesanías Tradicionales”. En Patrimonio, ciencia y comunidad. Su abordaje en los partidos de Azul, Olavarría y Tandil, editado por María Luz Endere y José Luis Prado, 351-364. Olavarría: Universidad Nacional del Centro de la Provincia de Buenos Aires.

Criado Aguilar, Encarnación. 2005. "Patrimonio y globalización: el recurso de la cultura en las políticas de desarrollo europeas”.
Cuadernos de Antropología Social. Cultura y Patrimonio, Perspectivas Contemporáneas en la Investigación y la Gestión 21: 51-69.

Dawson, Munjeri. 2004. "Patrimonio material e inmaterial: de la diferencia a la convergencia”. Museum Internacional. Intangible Heritage 221/222: 13-21.

Duguine, Laura. 2010. “Conflictos entre los colonos alemanes del Volga y el Estadonación: la conformación de una nación ‘civilizada'”. En Arqueología argentina en el bicentenario de la Revolución de Mayo, editado por Roberto Bárcena y Horacio Chiavazza, tomo II, 437-441. Mendoza: Universidad Nacional de Cuyo.

Endere, María Luz. 2014. "Indigenous Peoples' Rights in Latin America”. En Encyclopedia of Global Archaeology, editado por Claire Smith, 4441-4448. Nueva York: Springer.

Endere María Luz y José Luis Prado. 2009. “Criterios de selección, valoración y zonificación de yacimientos arqueológicos y paleontológicos”. En Patrimonio, ciencia y comunidad. Su abordaje en los partidos de Azul, Olavarría y Tandil, editado por María Luz Endere y José Luis Prado, 47-65. Olavarría: Universidad Nacional del Centro de la Provincia de Buenos Aires.

Endere, María Luz y Mercedes Mariano. 2013. "Los conocimientos tradicionales y los desafíos de su protección legal en Argentina”. Revista Quinto Sol 17 (2): 1-20.

García Canclini, Néstor. 1989. Culturas híbridas. Estrategias para entrar y salir de la modernidad. México: Grijalbo.

-. 1999. "Los usos sociales del patrimonio cultural”. En Patrimonio etnológico. Nuevas perspectivas de estudio, editado por Encarnación Aguilar Criado, 16-33. Sevilla: Consejería de la Cultura, Junta de Andalucía. 
Giménez, Gilberto. 2005. Teoría y análisis de la cultura (vol. I). México: Colección Intersecciones 5.

Giorgis, Marta. 2004. La Virgen prestamista. La fiesta de la Virgen de Urkupiña en el boliviano Gran Córdoba. Buenos Aires: Antropofagia.

Guber, Rosana. 2011. La etnografía. Método, campoy reflexibilidad. Buenos Aires: Siglo XXI.

Kirshenblatt-Gimblett, Bárbara. 2004. "El patrimonio inmaterial como producción metacultural". Museum Intencional. Intangible Heritage 221/222: 52-67.

Kurin, Richard. 2004. "La salvaguarda del patrimonio cultural inmaterial en la Convención de la Unesco del 2003: una valoración crítica”. Museum Internacional. Intangible Heritage 221/222: 68-81.

Lacarrieu, Mónica. 2010. “¿Es necesario patrimonializar las expresiones culturales ‘inmateriales'? Desafíos teóricos y metodológicos en torno del patrimonio cultural 'intangible'”. Actas en C. D. del $1{ }^{\text {er }}$ Congreso Iberoamericano sobre Patrimonio Cultural. San José: Universidad de Costa Rica.

\section{Lacarrieu, Mónica y Marcelo Álvarez.} 2008. "La plaza y la caverna. Dilemas contemporáneos de la gestión cultural”. En La (indi)gestión cultural. Una categoría de los procesos culturales contemporáneos, compilado por Mónica Lacarrieu y Marcelo Álvarez, 11-30. Buenos Aires: La Crujía.

\section{Ladrón de Guevara, Bernarda.}

2011. Estándares mínimos de registro del patrimonio arqueológico. Santiago de Chile: Centro Nacional de Conservación y restauración, Dibam; Consejo de Monumentos Nacionales, área del Sistema Nacional de Coordinación de Información Territorial.
Laidlaw, Rob. 1989. "Cultural Resource Planning and Management in a MultipleUse Agency". En Archaeological Heritage Management in the Modern World, editado por Henry Cleere, 232-235. Londres: Council of British Archaeology.

\section{Lima Filho, Manuel Ferreira y} Regina Maria do Rego Monteiro de Abreu. 2010. "La antropología y el patrimonio cultural en Brasil”. Revista Colombiana de Antropología 46 (1): 133-155.

Mariano, Carolina. 2013. "Desarrollo y aplicación de herramientas teóricometodológicas para la gestión sustentable del patrimonio arqueológico en el centro de la provincia de Buenos Aires, Argentina”. Tesis de doctorado en Arqueología, Facultad de Ciencias Sociales, Unicen, Olavarría, Buenos Aires.

Mariano, Mercedes y María Luz Endere. 2013. "Reflexiones acerca de la protección del patrimonio intangible a nivel internacional, regional y su proyección en Argentina”. Dimensión Antropológica 2 (58): 33-59.

Montenegro, Mauricio. 2010. "La patrimonialización como protección contra la mercantilización: paradojas de las sanciones culturales de lo igual y lo diferente". Revista Colombiana de Antropología 46 (1): 115-131.

National Center for Geographic Information and Analysis (NCGIA). 1990. What is GIS? Santa Bárbara: University of California.

Pedrotta, Victoria, Mariela Tancredi, Mercedes Mariano y María Luz Endere. 2013. “Tejiendo saberes. Patrimonio intangible, identidad y valoración social: el caso de Ercilia Cestac”. Revista Runa 1 (34): 91-112.

Prats, Llorenç. 2000. "El concepto de patrimonio cultural”. Cuadernos de Antropología Social 11: 115-135. 
-. 2007. Antropología y patrimonio. Barcelona: Ariel.

Rosas Mantecón, Ana. 1998. Introducción a Alteridades 16: 3-9.

Rotman, Mónica. 2004. "La creación urbana de tradiciones locales”. En Antropología de la cultura y el patrimonio. Diversidady desigualdad en los procesos contemporáneos, editado por Mónica Rotman, 135-151. Buenos Aires: Ferreyra.

Salge Ferro, Manuel. En prensa. "El patrimonio cultural inmaterial como principio de autoridad”. Boletín OPCA 6: 4-7.

Sánchez Carretero, Cristina. 2005. "Sobre el patrimonio inmaterial de la humanidad y la lucha por visibilizar 'lo africano’ en la República Dominicana”. En Patrimonio cultural: politizaciones y mercantilizaciones, coordinado por Xosé Carlos Sierra Rodríguez y Xerardo Pereiro Pérez, 147-163. Sevilla: FFAAEE.
Santoyo, Álvaro. 2006. Investigación para la definición de un marco conceptual de la política sobre patrimonio cultural en Colombia. Observatorio de Patrimonio Material, Inmaterial y Arqueológico (MIA). Bogotá: ICANH.

Unesco. 2003. Convención para la salvaguardia del patrimonio cultural inmaterial. Consultado el 28 de marzo del 2013. http:|www.unesco.org.

Van Maanen, John. 1995. “An End to Innocence: The Ethnography of Ethnography”. En Representation in Ethnography, editado por John van Maanen, 1-35. Londres: University College.

Van Zanten, Wim. 2004. "La elaboración de una nueva terminología para el patrimonio cultural inmaterial". Museum Internacional. Intangible Heritage 221/222: 36-43. 